\title{
Calculation of the need for paediatric intensive care beds
}

\author{
Eugene Milne, Paula Whitty
}

\begin{abstract}
A study of paediatric intensive care usage and need was undertaken in the former English Northern region to define appropriate local provision in the light of apparently conflicting published evidence. It was hypothesised that daily bed need would follow a Poisson distribution. All admissions of children aged less than 15 years who required intensive care in the region were recorded retrospectively for the financial year 1993/4. The mean number of beds occupied was 11.7 per day, which is equivalent to 20.7 beds per million children per day. The distribution of numbers of beds used mirrored a Poisson distribution closely, and the predicted bed requirement to cover $95 \%$ of days in the year was in agreement with that observed. Review of recommendations for paediatric intensive care provision from other studies suggests that apparent differences arise largely from the effect of different sizes of population served, and that, when allowance is made for this, underlying rates of bed requirement are strikingly similar, with a mean of around 20 per million children per day. A formula is given for the application of this model to local populations.

(Arch Dis Child 1995; 73: 505-507)
\end{abstract}

Keywords: paediatric intensive care, bed allocation, bed occupancy, intensive care provision.

Recent Department of Health guidance on paediatric intensive care provision has highlighted the need for figures on bed provision requirements, ${ }^{1}$ but there is no clear agreement on an appropriate number of beds for any given population.

The British Paediatric Association (BPA) in 1993 recommended one bed per 48000 children, ${ }^{2}$ on the basis of a survey of 500 wards and units admitting children. This was regarded as being similar to previously published estimates of need, notably the BPA's 1987 estimate of one bed per 40000 children, which had been based on American data. ${ }^{3}$

In 1992, $\mathrm{Yeh}^{4}$ proposed a requirement of roughly one bed per 28-32 000 children in the United States, while Shann in $1993^{5}$ proposed that the United Kingdom would require 12-14 paediatric intensive care units serving around one million children each, which has been interpreted by the $\mathrm{BPA}^{2}$ as implying a requirement of one bed per 60-80 000 children.

On the basis of Birmingham data for 1991, Barry and Hocking have recently suggested, however, that one bed per 26000 children would be required to cover demand for $95 \%$ of the year. ${ }^{6}$

In order to identify the local need for paediatric intensive care provision, a study was conducted of usage and need for intensive care facilities in the former Northern region, and is described fully in a 1994 report ( $P$ Whitty. Paediatric intensive care services. An assessment of need in the area covered by the former Northern region. Final draft report, unpublished).

In analysing these data we hypothesised that daily bed usage would follow a Poisson distribution, as the requirement for intensive care represents a rare event in a large population of children. ${ }^{7}$ The purpose of this paper is to test this hypothesis on the data obtained from the Northern region's study and to establish whether a standard formula can be used for calculating the needs of a given population.

\section{Methods}

All admissions of children aged 16 and under who required intensive care in the former Northern region were recorded retrospectively for the financial year 1993/94. This was done by postal questionnaire and telephone inquiries to all facilities likely to admit children requiring intensive care. The criteria for inclusion in the study were:

- Mechanical ventilatory support or endotracheal intubation (except in the very short term)

- Continuous invasive monitoring of arterial, central venous or intracranial pressure

- Vasoactive drug infusion (such as dopamine or dobutamine).

Where this information was not available but the admission was to a recognised intensive care unit, the admission was included in the study. Thus a database was assembled that contained details of admission and discharge dates for all children in this age group treated in, or requiring treatment in, intensive care facilities.

For the purposes of this paper, only the data on children aged under 15 years were used to enable comparison with the Birmingham data. ${ }^{6}$

Bed occupancy on each day was calculated as in the Birmingham study ${ }^{6}$ by adding together the numbers of admissions, day cases, and beds occupied over the preceding night, then subtracting the number of discharges for that day. Discharge dates falling after the end of the study year had 365 days subtracted from them to allow for those patients admitted before, but discharged during, the study year. 
Frequency and cumulative percentage of days on which given number of beds were occupied in the Northern region 1993/4

\begin{tabular}{lcc}
\hline No of beds & $\begin{array}{l}\text { Frequency of days on } \\
\text { which beds occupied }\end{array}$ & Cumulative \% \\
\hline 1 & 1 & $0 \cdot 3$ \\
2 & 0 & $0 \cdot 3$ \\
3 & 0 & $0 \cdot 3$ \\
4 & 1 & $0 \cdot 5$ \\
5 & 5 & $1 \cdot 9$ \\
6 & 13 & $5 \cdot 5$ \\
7 & 31 & $14 \cdot 0$ \\
8 & 36 & $23 \cdot 8$ \\
9 & 35 & $33 \cdot 4$ \\
10 & 37 & $43 \cdot 6$ \\
11 & 34 & $52 \cdot 9$ \\
12 & 34 & $62 \cdot 2$ \\
13 & 24 & $68 \cdot 8$ \\
14 & 26 & $75 \cdot 9$ \\
15 & 25 & $82 \cdot 7$ \\
16 & 12 & $86 \cdot 0$ \\
17 & 21 & $91 \cdot 8$ \\
18 & 16 & $96 \cdot 2$ \\
19 & 4 & $97 \cdot 3$ \\
20 & 4 & $98 \cdot 4$ \\
21 & 4 & $99 \cdot 5$ \\
22 & 0 & $99 \cdot 5$ \\
23 & 1 & $99 \cdot 7$ \\
24 & 1 & 100 \\
Total & 365 & \\
\hline
\end{tabular}

This method yields a figure for each day of the year relative to the number of beds occupied overnight on 31 March 1993, a baseline figure which was calculated to be 14 occupied beds. The accuracy of the calculated bed occupancy figures was checked against days of the year on which the starting conditions were reproduced and found to be precisely right in all instances. There were 542 cases with both an admission and a discharge date, while 21 cases, having admission dates only, were assigned the median length of stay. There were no cases continually occupying intensive care beds throughout the entire period of study.

Denominator population figures were the 1991 based final mid-year population estimates for 1993 for the former Northern region. ${ }^{8}$

The mean number of beds occupied per day was used to calculate Poisson centiles, and provide an estimate of beds required for comparison with the observed figure.

All analyses were performed using Epi Info version $6 .{ }^{9}$

\section{Results}

During 1993-94 there were 563 episodes of intensive care provision for children aged less than 15. Bed occupancy per day is illustrated in the table. The mean number of beds occupied was 11.7 per day, which is equivalent to 20.7 beds per million children per day, based on the 1993 population estimate for the region of 563000 children aged less than 15 years. Using 11.7 as the estimate of population mean, calculation of the 95th centile of the poisson distribution suggested that 18 beds would have accommodated demand on $95 \%$ of days in the year. In practice, this number would have been sufficient on $96 \cdot 2 \%$ of days.

Figure 1 shows the number of days in the study year that would have been accommodated by given numbers of paediatric intensive care beds in a single unit, both for our data and for those from Birmingham. For comparison, the predicted curves based on Poisson distributions about the observed mean bed occupancies are given as broken lines.

\section{Discussion}

Describing bed requirements in terms of $n$ per million population implicitly assumes a linear relationship between the bed requirement and the population served. As the requirement for intensive care is a rare event in a large population, we suggest that the most likely theoretical model for bed usage is of a Poisson distribution about the mean number of beds occupied per day. Under this model, the relationship between beds required to accommodate all patients on a given proportion of days and the population served is a curve, such that small populations will have a higher value of $n$ than larger populations.

This is shown in fig 2, where for a population of 100000 with a mean bed occupancy per day of 20 beds per million, four beds would be required to accommodate need on $95 \%$ of days in the year (one per 25000 ), while for a population of one million only 27 would be required (one per 37000 ) rather than the 40 that might be expected in a linear relationship.

Two basic assumptions must be made in adopting this hypothesis; firstly that the underlying rate of bed occupancy is constant, and secondly, that deviations in bed occupancy from day to day are statistically independent. Neither of these assumptions is likely to be entirely true, given that there will be some seasonal variation in bed occupancy, and that successive days will be correlated because of overnight and longer stays. The net effect of these deviations from the model would be to render the variance greater than the mean, rather than being equal to it as would be expected in a Poisson distribution. This is true for our figures, being manifest as a slight flattening of the actual versus theoretical curves in fig 1, and accounting for the marginal underestimate of bed requirements yielded by the model both in our data and in the Birmingham figures. ${ }^{6}$ In our figures the difference does not alter the predicted bed requirement, while for Birmingham the addition of

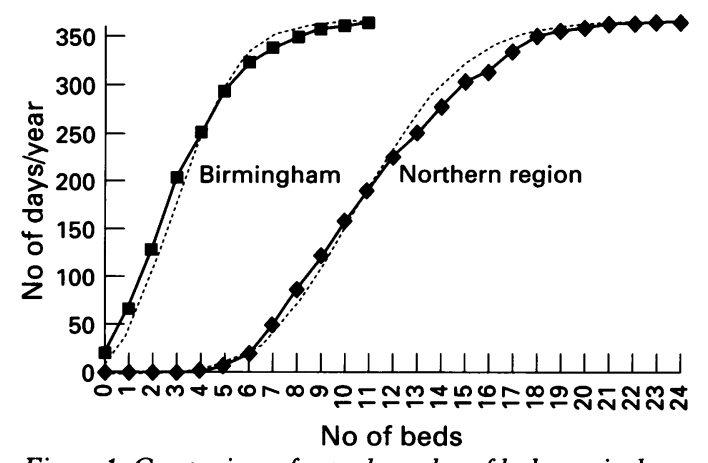

Figure 1 Comparison of actual number of beds required to accommodate intensive care unit need against days in the year, for Birmingham and Northern region data. Broken lines represent bed requirement predicted by Poisson distribution about the observed mean number of beds occupied per day in each study. 


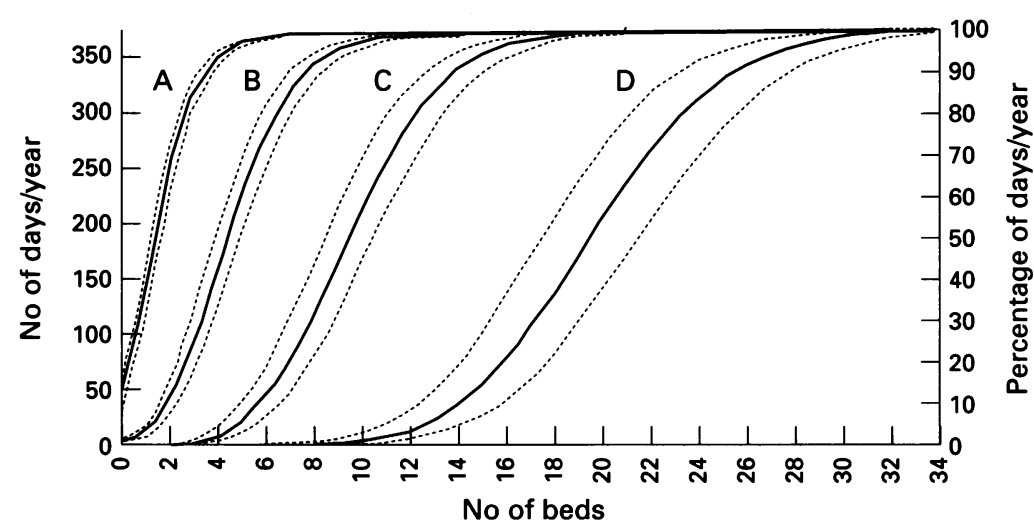

Figure 2 Poisson based bed numbers required ( $x$ axis) to satisfy requirements for varying proportions of the year (y axes) at four different sizes of population $(A, 100000 ; B$, 250 000; $C, 500000 ; D, 1000000$ ), with provision at 20 beds per million children per day (solid lines) and at 18 and 22 beds per million children per day (left and right broken lines respectively). Assuming that we wish to accomodate demand on 95\% (347) of days of the year, a horizontal line can be drawn across the graph at that level on the $y$ axes. This will intersect each of the solid lines $A, B, C$, and $D$ at points vertically above the bed numbers needed, as indicated on the $x$ axis. Line $A$ represents a population of 100000 and the intersection point is at 4 beds, whereas line $D$ represents a population of 1000000 and the intersection is at 27 beds. If there was a linear relationship between population and bed requirement, these figures would have a ratio of 1:10 rather than the observed 1:7. The ranges illustrated with broken lines show the variation in bed numbers required that would be brought about by using different estimates of the mean number of beds per million children per day - in this case 18 (left broken line) and 22 (right broken line).

one extra bed more than compensates for this discrepancy.

Thus in the Birmingham study the mean number of beds occupied was 3.7 per day, which is equivalent to $17 \cdot 17$ per million children per day. Using this estimate of the mean for a population of this size places the 95th centile of the Poisson distribution at 7 beds. Comparison with the actual requirement shows that this number of beds would have been sufficient on $93 \%$ of days in the year, and the addition of one more bed would have raised the figure to $96 \%$.

Clearly, the Poisson based estimate of required beds is appropriate for both our data and those from Birmingham, but, importantly, the mean numbers of beds occupied per day $(17 \cdot 17 v 20 \cdot 7)$ are similar. Even more striking is the similarity between the figure derived from the BPA survey, 20.9 beds per million population, ${ }^{2}$ and our own. It should be borne in mind that the BPA looked at children aged 16 and under, rather than under 15 as described here, but this is unlikely to have made a large difference to the rate of bed occupancy per million. These different studies are thus consistent in their findings, despite the apparent difference suggested by the manner of their presentation. What difference does exist may be accounted for by the inclusion criteria for the studies. Although the same in principle (the Northern region study criteria were based on those used in Birmingham), incompleteness of information in our study may have led to the inclusion of some ineligible cases, whereas the criteria could be applied more strictly in Birmingham as medical notes were traced.

Shann's estimate ${ }^{5}$ of United Kingdom paediatric intensive care need, which suggested that the admission rate for a catchment population of one million children would be around 1200 cases per year, is consistent with Northern region figures. His further suggestion, that these could be accommodated in 14-16 beds, given a Poisson distribution, would require a much shorter mean length of stay than we have observed. This is consistent with Shann's observation elsewhere ${ }^{10}$ that United Kingdom paediatric intensive care units currently run at low rates of bed occupancy, and could be made more efficient through shorter lengths of stay.

Yeh's estimate ${ }^{4}$ of one bed per 28-32 000, was based on 10 units serving an average of 300000 children. For a unit of this size, with a mean daily bed occupancy of 20 beds per million children, $95 \%$ of days in the year would be accommodated by 11 beds under the Poisson model, representing in this population one bed per 27000 children, an estimate strikingly similar to that observed.

We conclude, therefore, that an appropriate level of provision in a single unit to accommodate need on $95 \%$ of days in the year can be calculated by taking the 95 th centile of a Poisson distribution about the mean number of beds required per day. This can be derived simply for any population $(P)$ by using the formula:

$$
\begin{gathered}
\text { Bed requirement }=m+1 \cdot 64 \sqrt{m} \\
\text { where } m \pm P \times 20 / 1000000
\end{gathered}
$$

provided the value of $m$ is not too small. Consideration would need to be given to a small adjustment upwards to compensate for the anticipated deviation from the model described above, bearing in mind that an additional bed at this level of coverage will be used for less than one month in the year.

Analysis of existing guidance using this model suggests that there is a greater degree of consistency in recommendations for intensive care provision than is apparent at first sight. While this does not necessarily imply that 20 beds per million children per day is the correct level of provision, this approach provides a more rational basis upon which to conduct that debate.

1 EL(94)10. London: Department of Health, 1994

2 British Paediatric Association. The care of critically ill children - report of a multidisciplinary working party of intensive care. London: BPA, 1993.

3 British Paediatric Association. Report of a working party on paediatric intensive care. London: BPA, 1987.

4 Yeh TS. Regionalisation of pediatric critical care. Crit Care Clin 1992; 8: 23-35.

5 Shann F. Australian view of paediatric intensive care in Britain. Lancet 1993; 342: 68.

6 Barry PW, Hocking MD. Paediatric use of intensive care. Arch Dis Child 1994; 70: 391-4.

7 Armitage P, Berry G. Statistical methods in medical research. 2nd Ed. Oxford: Blackwell, 1987.

8 Office of Population Censuses and Surveys. Final rebased population estimates 1991. London: OPCS, 1993.

9 Dean AG, Dean JA, Couilombier D, et al. Epi Info version 6: a word processing, database, and statistics program for epidemiology on microcomputers. Atlanta: Centers for Disease Control and Prevention, 1994.

10 Shann F. In: $A$ critical appraisal of the care of critically ill children and implications for policy, research, and development. York: NHS Centre for Reviews and Dissemination, 1994. 